DOI 10.4171/JEMS/207

Xiaoli Han · Jiayu Li

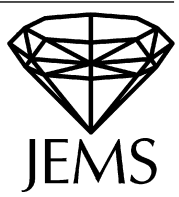

\title{
Symplectic critical surfaces in Kähler surfaces
}

Received December 18, 2007 and in revised form May 29, 2008

\begin{abstract}
Let $M$ be a Kähler surface and $\Sigma$ be a closed symplectic surface which is smoothly immersed in $M$. Let $\alpha$ be the Kähler angle of $\Sigma$ in $M$. We first deduce the Euler-Lagrange equation of the functional $L=\int_{\Sigma} \frac{1}{\cos \alpha} d \mu$ in the class of symplectic surfaces. It is $\cos ^{3} \alpha H=$ $\left(J(J \nabla \cos \alpha)^{\top}\right)^{\perp}$, where $H$ is the mean curvature vector of $\Sigma$ in $M$, and $J$ is the complex structure compatible with the Kähler form $\omega$ in $M$; it is an elliptic equation. We call a surface satisfying this equation a symplectic critical surface. We show that, if $M$ is a Kähler-Einstein surface with nonnegative scalar curvature, each symplectic critical surface is holomorphic. We also study the topological properties of symplectic critical surfaces. By our formula and Webster's formula, we deduce that the Kähler angle of a compact symplectic critical surface is constant, which is not true for noncompact symplectic critical surfaces.
\end{abstract}

Keywords. Symplectic surface, holomorphic curve, Kähler surface

\section{Introduction}

Suppose that $M$ is a Kähler surface. Let $\omega$ be the Kähler form on $M$ and let $J$ be a complex structure compatible with $\omega$. The Riemannian metric $\langle$,$\rangle on M$ is defined by

$$
\langle U, V\rangle=\omega(U, J V) .
$$

For a compact oriented real surface $\Sigma$ which is smoothly immersed in $M$, one defines, following [4], the Kähler angle $\alpha$ of $\Sigma$ in $M$ by

$$
\left.\omega\right|_{\Sigma}=\cos \alpha d \mu_{\Sigma}
$$

where $d \mu_{\Sigma}$ is the area element of $\Sigma$ of the metric induced from $\langle$,$\rangle . We say that \Sigma$ is a holomorphic curve if $\cos \alpha \equiv 1 ; \Sigma$ is a Lagrangian surface if $\cos \alpha \equiv 0$, and a symplectic surface if $\cos \alpha>0$.

Xiaoli Han: Department of Mathematical Sciences, Tsinghua University, Beijing 100084,

P. R. China; e-mail: xlhan@math.tsinghua.edu.cn

Jiayu Li: Math. Group, The Abdus Salam ICTP, Trieste 34100, Italy and Academy of Mathematics and Systems Sciences, Chinese Academy of Sciences, Beijing 100080, P. R. China; e-mail: jyli@ictp.it

Mathematics Subject Classification (2010): Primary 53C42; Secondary 58J05 
It was conjectured by Tian [8] that every embedded orientable closed symplectic surface in a compact Kähler-Einstein surface is isotopic to a symplectic minimal surface in a suitable sense.

If the Kähler-Einstein surface is of nonnegative scalar curvature, a symplectic minimal surface is holomorphic. However, if the scalar curvature is negative, there are symplectic minimal surfaces which are not holomorphic ([1]). A symplectic minimal surface is a critical point of the area of surfaces, which is symplectic. It may be more natural to consider directly the critical point of the functional

$$
L=\int_{\Sigma} \frac{1}{\cos \alpha} d \mu_{\Sigma}
$$

in the class of symplectic surfaces in a Kähler surface. It is clear that holomorphic curves minimize the functional. A critical point of this functional is called a symplectic critical surface.

In the paper, we first calculate the Euler-Lagrange equation of the functional $L$.

Theorem 1.1. Let $M$ be a Kähler surface. The Euler-Lagrange equation of the functional $L$ is

$$
\cos ^{3} \alpha H-\left(J(J \nabla \cos \alpha)^{\top}\right)^{\perp}=0,
$$

where $H$ is the mean curvature vector of $\Sigma$ in $M,()^{\top}$ is the tangential component of (), and ()$^{\perp}$ is the normal components of ().

We will check that the above equation is elliptic. We also derive an equation for the Kähler angle of a symplectic critical surface in a Kähler-Einstein surface.

Theorem 1.2. If $M$ is a Kähler-Einstein surface and $\Sigma$ is a symplectic critical surface, then

$$
\Delta \cos \alpha=\frac{3 \sin ^{2} \alpha-2}{\cos \alpha}|\nabla \alpha|^{2}-K_{0} \cos ^{3} \alpha \sin ^{2} \alpha,
$$

where $K_{0}$ is the scalar curvature of $M$.

As a corollary, we see that, if the scalar curvature $K_{0}$ of the Kähler-Einstein surface $M$ is nonnegative, then a symplectic critical surface in $M$ is holomorphic.

It is not difficult to see that a nonholomorphic symplectic critical surface in a Kähler surface has at most finitely many complex points. Moreover, we can show:

Theorem 1.3. Suppose that $\Sigma$ is a nonholomorphic symplectic critical surface in a Kähler surface M. Then

$$
\chi(\Sigma)+\chi(\nu)=-P-\frac{1}{2 \pi} \int_{\Sigma} \frac{|\nabla \alpha|^{2}}{\cos ^{2} \alpha} d \mu,
$$

and

$$
c_{1}(M)([\Sigma])=-P-\frac{1}{2 \pi} \int_{\Sigma} \frac{|\nabla \alpha|^{2}}{\cos ^{3} \alpha} d \mu,
$$

where $\chi(\Sigma)$ is the Euler characteristic of $\Sigma, \chi(v)$ is the Euler characteristic of the normal bundle of $\Sigma$ in $M, c_{1}(M)$ is the first Chern class of $M,[\Sigma] \in H_{2}(M, \mathbb{Z})$ is the homology class of $\Sigma$ in $M$, and $P$ is the number of complex tangent points. 
From Theorem 1.3, and Webster's formula ([9, Proposition 1]), we have the following theorem.

Theorem 1.4. Suppose that $\Sigma$ is a symplectic critical surface in a Kähler surface $M$. Then

$$
\chi(\Sigma)+\chi(v)=c_{1}(M)([\Sigma])
$$

and $\Sigma$ is a minimal surface with constant Kähler angle.

If $\Sigma$ is not compact, the theorem is not true. The rotational symmetric surface $z=$ $-\frac{1}{2} \log \left(x^{2}+y^{2}\right)$ in $\mathbb{C}^{2}$ is a symplectic critical surface which is not minimal.

Recall that a minimal surface with constant Kähler angle is an infinitesimally holomorphic immersion introduced by Micallef-Wolfson [6]. The theorem shows that we may use the variation of the functional $L$ to find infinitesimally holomorphic immersions which are holomorphic in many cases.

Let $g$ be the genus of $\Sigma, I_{\Sigma}$ the self-intersection number of $\Sigma$, and $D_{\Sigma}$ the number of double points of $\Sigma$. Then

$$
\chi(\Sigma)=2-2 g, \quad \chi(v)=I_{\Sigma}-2 D_{\Sigma} .
$$

Setting

$$
c_{1}(\Sigma)=c_{1}(M)([\Sigma])
$$

we have

Theorem 1.5. Suppose that $\Sigma$ is a symplectic critical surface in a Kähler surface $M$. Then

$$
2-2 g-c_{1}(\Sigma)+I_{\Sigma}-2 D_{\Sigma}=0 .
$$

In forthcoming papers, we will use a variational approach and the flow method to study the existence of symplectic critical surfaces in a Kähler surface.

It is natural to conjecture that, in each homotopy class of symplectic surfaces in a Kähler surface, there is a symplectic critical surface.

As a starting point for the study of the gradient flow of the function $L$, we derive the evolution equation of $\cos \alpha$ along the flow, which implies that the symplectic property is preserved.

\section{The Euler-Lagrange equation}

Let $\left\{\phi_{t}\right\}_{0 \leq t \leq 1}$ be a one-parameter family of immersions $\Sigma \rightarrow M$ such that $\phi_{0}=F$ and $\Sigma_{t}=\phi_{t}(\Sigma)$ are symplectic. Also, let $X$ denote the initial velocity vector for $\phi_{t}$, i.e, $X=\left.\frac{\partial \phi_{t}}{\partial t}\right|_{t=0}$. We denote by $\bar{\nabla}$ the covariant derivative and by $K$ the Riemannian curvature tensor on $M$. Furthermore, $\nabla, R$ denote the covariant derivative and the Riemannian curvature tensor of the induced metric $g$ on the surface $\Sigma_{0}$.

We start by computing the first variation of the area for this one-parameter family of surfaces, which is in fact well-known. 
Proposition 2.1. The variation of the area of $\Sigma_{t}$ is

$$
\left.\frac{\partial}{\partial t} d \mu_{t}\right|_{t=0}=\left(\operatorname{div} X^{\top}-X \cdot H\right) d \mu_{t}
$$

Proof. The proof is routine (cf. [7]). Fix $p \in \Sigma$. Let $\left\{x^{i}\right\}$ be a normal coordinate system for $\Sigma$ at $p$. Around $p$ we choose a local orthonormal frame $\left\{\tilde{e}_{1}, \tilde{e}_{2}, \tilde{v}_{3}, \tilde{v}_{4}\right\}$ on $M$ along $\Sigma_{t}$ such that $\left\{\tilde{e}_{1}=\partial \phi_{t} / \partial x^{i}, \tilde{e}_{2}=\partial \phi_{t} / \partial x^{2}\right\}$ and $\left\{\tilde{v}_{3}, \tilde{v}_{4}\right\}$ are in the tangent bundle and in the normal bundle of $\Sigma_{t}$ respectively. For simplicity, we denote $\tilde{e}_{i}(0)=\partial F / \partial x^{i}$ by $e_{i}$ and identify it with $\partial_{i}=\partial / \partial x^{i}, i=1,2$. We also denote $\tilde{v}_{\alpha}(0)$ by $v_{\alpha}, \alpha=3,4$. Furthermore, we assume that $\nabla_{e_{i}} e_{j}=0$ at $p$. Suppose that in this frame $X$ takes the form $X=X^{i} e_{i}+X^{\alpha} v_{\alpha}$ and $\left(g_{t}\right)_{i j}=\left\langle\partial \phi_{t} / \partial x^{i}, \partial \phi_{t} / \partial x^{j}\right\rangle$. Then

$$
\begin{aligned}
\left.\frac{\partial g_{i j}}{\partial t}\right|_{t=0} & =\left.\frac{\partial}{\partial t}\right|_{t=0}\left\langle\partial \phi_{t} / \partial x^{i}, \partial \phi_{t} / \partial x^{j}\right\rangle=\left\langle\bar{\nabla}_{e_{i}} X, e_{j}\right\rangle+\left\langle e_{i}, \bar{\nabla}_{e_{j}} X\right\rangle \\
& =\left\langle\bar{\nabla}_{e_{i}}\left(X^{k} e_{k}+X^{\alpha} v_{\alpha}\right), e_{j}\right\rangle+\left\langle e_{i}, \bar{\nabla}_{e_{j}}\left(X^{k} e_{k}+X^{\alpha} v_{\alpha}\right)\right\rangle \\
& =X_{j, i}-X_{\alpha} h_{i j}^{\alpha}+X_{i, j}-X_{\alpha} h_{i j}^{\alpha} .
\end{aligned}
$$

It is easy to see that

$$
\left.\frac{\partial}{\partial t} d \mu_{t}\right|_{t=0}=\frac{1}{2} g^{i j}\left(X_{j, i}-X_{\alpha} h_{i j}^{\alpha}+X_{i, j}-X_{\alpha} h_{i j}^{\alpha}\right) d \mu_{t}=\left(\operatorname{div} X^{\top}-X \cdot H\right) d \mu .
$$

Theorem 2.2. Let $M$ be a Kähler surface. The first variational formula of the functional $L$ is, for any smooth vector field $X$ on $\Sigma$,

$$
\delta_{X} L=-2 \int_{\Sigma} \frac{X \cdot H}{\cos \alpha} d \mu+2 \int_{\Sigma} \frac{\left.X \cdot\left(J(J \nabla \cos \alpha)^{\top}\right)\right)^{\perp}}{\cos ^{4} \alpha} d \mu,
$$

where $H$ is the mean curvature vector of $\Sigma$ in $M,()^{\top}$ is the tangential component of (), and ()$^{\perp}$ is the normal component of (). The Euler-Lagrange equation of the functional $L$ is

$$
\cos ^{3} \alpha H-\left(J(J \nabla \cos \alpha)^{\top}\right)^{\perp}=0 .
$$

Proof. We use the frame that we have chosen in Proposition 2.1. From the definition of Kähler angle (1.1) we have

$$
\cos \alpha_{t}=\frac{\omega\left(\partial \phi_{t} / \partial x^{1}, \partial \phi_{t} / \partial x^{2}\right)}{\sqrt{\operatorname{det}\left(g_{t}\right)}}
$$

where $\operatorname{det}\left(g_{t}\right)$ is the determinant of the metric $\left(g_{t}\right)$. So, the functional can be written as

$$
L_{t}=L\left(\phi_{t}\right)=\int_{\Sigma} \frac{\operatorname{det}\left(g_{t}\right)}{\omega\left(\partial \phi_{t} / \partial x^{1}, \partial \phi_{t} / \partial x^{2}\right)} d x^{1} \wedge d x^{2} .
$$


Thus using Proposition 2.1 we have

$$
\begin{aligned}
\left.\frac{d}{d t}\right|_{t=0} L_{t}= & \int_{\Sigma}\left(\frac{\left.\partial_{t} g_{i j}\right|_{t=0} g^{i j}}{\omega\left(e_{1}, e_{2}\right)}-\frac{\left.\partial_{t} \omega\left(\partial \phi_{t} / \partial x^{1}, \partial \phi_{t} / \partial x^{2}\right)\right|_{t=0}}{\omega^{2}\left(e_{1}, e_{2}\right)}\right) \operatorname{det}(g) d x^{1} \wedge d x^{2} \\
= & \int_{\Sigma} \frac{2 \operatorname{div} X^{\top}-2 X \cdot H}{\cos \alpha} \sqrt{\operatorname{det}(g)} d x^{1} \wedge d x^{2} \\
& -\int_{\Sigma} \frac{\omega\left(\bar{\nabla}_{e_{1}} X, e_{2}\right)+\omega\left(e_{1}, \bar{\nabla}_{e_{2}} X\right)}{\cos ^{2} \alpha} d x^{1} \wedge d x^{2} \\
= & I+I I .
\end{aligned}
$$

Since $\Sigma$ is closed, applying the Stokes formula, we obtain

$$
I=\int_{\Sigma}\left(\frac{2\left\langle X, e_{1}\right\rangle \nabla_{e_{1}} \cos \alpha+2\left\langle X, e_{2}\right\rangle \nabla_{e_{2}} \cos \alpha}{\cos ^{2} \alpha}-\frac{2 X \cdot H}{\cos \alpha}\right) \sqrt{\operatorname{det}(g)} d x^{1} \wedge d x^{2} .
$$

The second term is

$$
\begin{aligned}
I I= & -\int_{\Sigma} \frac{\nabla e_{1}\left(\omega\left(X, e_{2}\right)\right)-\omega\left(X, \bar{\nabla}_{e_{1}} \bar{\nabla}_{e_{2}} F\right)}{\cos ^{2} \alpha} d x^{1} \wedge d x^{2} \\
& -\int_{\Sigma} \frac{\nabla_{e_{2}}\left(\omega\left(e_{1}, X\right)\right)-\omega\left(\bar{\nabla}_{e_{2}} \bar{\nabla}_{e_{1}} F, X\right)}{\cos ^{2} \alpha} d x^{1} \wedge d x^{2} \\
= & -\int_{\Sigma} \frac{\nabla_{e_{1}}\left(\omega\left(X, e_{2}\right)\right)+\nabla_{e_{2}}\left(\omega\left(e_{1}, X\right)\right)}{\cos ^{2} \alpha} d x^{1} \wedge d x^{2} \\
= & -2 \int_{\Sigma} \frac{\omega\left(X, e_{2}\right) \nabla_{e_{1}} \cos \alpha+\omega\left(e_{1}, X\right) \nabla_{e_{2}} \cos \alpha}{\cos ^{3} \alpha} d x^{1} \wedge d x^{2},
\end{aligned}
$$

where we have used the fact that $\omega$ is parallel. In the following, we compute pointwise so we assume the frame is orthonormal. Note that

$$
\begin{aligned}
& \omega\left(X^{\top}, e_{2}\right)=-\left\langle X^{\top}, J e_{2}\right\rangle=-\left\langle X, e_{1}\right\rangle\left\langle e_{1}, J e_{2}\right\rangle=\left\langle X, e_{1}\right\rangle \cos \alpha, \\
& \omega\left(e_{1}, X^{\top}\right)=\left\langle X^{\top}, J e_{1}\right\rangle=\left\langle X, e_{2}\right\rangle\left\langle e_{2}, J e_{1}\right\rangle=\left\langle X, e_{2}\right\rangle \cos \alpha .
\end{aligned}
$$

We separate the second term into two parts,

$$
\begin{aligned}
I I= & -2 \int_{\Sigma}\left(\frac{\left\langle X, e_{1}\right\rangle \nabla_{e_{1}} \cos \alpha}{\cos ^{2} \alpha}+\frac{\left\langle X, e_{2}\right\rangle \nabla_{e_{2}} \cos \alpha}{\cos ^{2} \alpha}\right) d \mu \\
& -2 \int_{\Sigma} \frac{\omega\left(X^{\perp}, e_{2}\right) \nabla_{e_{1}} \cos \alpha+\omega\left(e_{1}, X^{\perp}\right) \nabla_{e_{2}} \cos \alpha}{\cos ^{3} \alpha} d \mu .
\end{aligned}
$$

Therefore, we obtain

$$
\begin{aligned}
\left.\frac{d}{d t}\right|_{t=0} L_{t}= & -2 \int_{\Sigma} \frac{X \cdot H}{\cos \alpha} d \mu-2 \int_{\Sigma} \frac{\omega\left(X^{\perp}, e_{2}\right) \nabla_{e_{1}} \cos \alpha}{\cos ^{3} \alpha} d \mu \\
& -2 \int_{\Sigma} \frac{\omega\left(e_{1}, X^{\perp}\right) \nabla_{e_{2}} \cos \alpha}{\cos ^{3} \alpha} d \mu .
\end{aligned}
$$


Because

$$
\begin{aligned}
(J \nabla \cos \alpha)^{\top} & =\left(J e_{1} \nabla_{e_{1}} \cos \alpha+J e_{2} \nabla_{e_{2}} \cos \alpha\right)^{\top} \\
& =\left\langle J e_{1}, e_{2}\right\rangle e_{2} \nabla_{e_{1}} \cos \alpha+\left\langle J e_{2}, e_{1}\right\rangle e_{1} \nabla_{e_{2}} \cos \alpha \\
& =\left(e_{2} \nabla_{e_{1}} \cos \alpha-e_{1} \nabla_{e_{2}} \cos \alpha\right) \cos \alpha,
\end{aligned}
$$

we have

$$
\begin{aligned}
\left.\frac{d}{d t}\right|_{t=0} L_{t} & =-2 \int_{\Sigma} \frac{X \cdot H}{\cos \alpha} d \mu-2 \int_{\Sigma} \frac{\omega\left(X^{\perp},(J \nabla \cos \alpha)^{\top}\right)}{\cos ^{4} \alpha} d \mu \\
& =-2 \int_{\Sigma} \frac{X \cdot H}{\cos \alpha} d \mu+2 \int_{\Sigma} \frac{\left.X \cdot\left(J(J \nabla \cos \alpha)^{\top}\right)\right)^{\perp}}{\cos ^{4} \alpha} d \mu .
\end{aligned}
$$

This completes the proof of the theorem.

For a later purpose, and to understand the equation, we express $\left.\left(J(J \nabla \cos \alpha)^{\top}\right)\right)^{\perp}$ at a fixed point $p$ in a local frame. Let $\left\{e_{1}, e_{2}, v_{3}, v_{4}\right\}$ be an orthonormal frame around $p \in \Sigma$ that is normal at $p$ and such that $\omega, J$ take the forms (cf. [3], [4])

$$
\omega=\cos \alpha u_{1} \wedge u_{2}+\cos \alpha u_{3} \wedge u_{4}+\sin \alpha u_{1} \wedge u_{3}-\sin \alpha u_{2} \wedge u_{4}
$$

where $\left\{u_{1}, u_{2}, u_{3}, u_{4}\right\}$ is the dual frame of $\left\{e_{1}, e_{2}, v_{3}, v_{4}\right\}$, and

$$
J=\left(\begin{array}{cccc}
0 & \cos \alpha & \sin \alpha & 0 \\
-\cos \alpha & 0 & 0 & -\sin \alpha \\
-\sin \alpha & 0 & 0 & \cos \alpha \\
0 & \sin \alpha & -\cos \alpha & 0
\end{array}\right)
$$

Then

$$
\begin{aligned}
\left.\left(J(J \nabla \cos \alpha)^{\top}\right)\right)^{\perp} & =\left(J\left(\cos \alpha \partial_{1} \cos \alpha e_{2}-\cos \alpha \partial_{2} \cos \alpha e_{1}\right)\right)^{\perp} \\
& =-\cos \alpha \sin \alpha \partial_{1} \cos \alpha v_{4}-\cos \alpha \sin \alpha \partial_{2} \cos \alpha v_{3} \\
& =\cos \alpha \sin ^{2} \alpha \partial_{1} \alpha v_{4}+\cos \alpha \sin ^{2} \alpha \partial_{2} \alpha v_{3} .
\end{aligned}
$$

Furthermore,

$$
\begin{aligned}
\partial_{1} \cos \alpha & =\omega\left(\bar{\nabla}_{e_{1}} e_{1}, e_{2}\right)+\omega\left(e_{1}, \bar{\nabla}_{e_{1}} e_{2}\right)=h_{11}^{\alpha}\left\langle J v_{\alpha}, e_{2}\right\rangle+h_{12}^{\alpha}\left\langle J e_{1}, v_{\alpha}\right\rangle \\
& =\left(h_{11}^{4}+h_{12}^{3}\right) \sin \alpha .
\end{aligned}
$$

Similarly, we can get

$$
\partial_{2} \cos \alpha=\left(h_{22}^{3}+h_{12}^{4}\right) \sin \alpha .
$$

Set $V=\partial_{2} \alpha v_{3}+\partial_{1} \alpha v_{4}$. Then

$$
V=-\left(h_{22}^{3}+h_{12}^{4}\right) v_{3}-\left(h_{11}^{4}+h_{12}^{3}\right) v_{4} .
$$

Consequently, the Euler-Lagrange equation of the functional $L$ is

$$
\cos ^{2} \alpha H-\sin ^{2} \alpha V=0,
$$


from which we see that $H=\sin \alpha \cdot g$ and $g$ is a smooth vector field on $N \Sigma$, or equivalently,

$$
H-\sin ^{2} \alpha(H+V)=0 .
$$

It is not difficult to see that, roughly, the symbol of the equation is

$$
\sigma:=\left(\begin{array}{cc}
\left(1-\sin ^{2} \alpha\right) \xi^{2}+\eta^{2} & \left(\sin ^{2} \alpha\right) \xi \eta \\
\left(\sin ^{2} \alpha\right) \xi \eta & \xi^{2}+\left(1-\sin ^{2} \alpha\right) \eta^{2}
\end{array}\right),
$$

which makes one believe that the equation 2.2 is elliptic.

In the following, we give a detailed proof.

Theorem 2.3. The equation (2.2) is elliptic.

Proof. Assume that $\Sigma$ is immersed in $M$ by $F$. Let $\{x, y\}$ be a coordinate system around $p \in \Sigma$. Since $\Sigma$ is smooth, by the implicit function theorem we can write $\Sigma$ as the graph of two functions $f, g$ in a small neighborhood $U$ of $p$, i.e., $F=(x, y, f(x, y), g(x, y))$ in $U$. Suppose that the complex structure in the neighborhood of $F(p)$ is standard, i.e.,

$$
J=\left(\begin{array}{cccc}
0 & -1 & 0 & 0 \\
1 & 0 & 0 & 0 \\
0 & 0 & 0 & -1 \\
0 & 0 & 1 & 0
\end{array}\right)
$$

We choose $e_{1}=\partial F / \partial x=\left(1,0, f_{x}, g_{x}\right), e_{2}=\partial F / \partial y=\left(0,1, f_{y}, g_{y}\right)$ and $v_{3}=$ $\left(-f_{x},-f_{y}, 1,0\right), v_{4}=\left(-g_{x},-g_{y}, 0,1\right)$. Then $\left\{e_{1}, e_{2}, v_{3}, v_{4}\right\}$ is a basis of $M$. The metric of $\Sigma$ in this basis is

$$
\left(g_{i j}\right)_{1 \leq i, j \leq 2}=\left(\begin{array}{ll}
1+f_{x}^{2}+g_{x}^{2} & f_{x} f_{y}+g_{x} g_{y} \\
f_{x} f_{y}+g_{x} g_{y} & 1+f_{y}^{2}+g_{y}^{2}
\end{array}\right)
$$

and the inverse matrix is

$$
\left(g^{i j}\right)_{1 \leq i, j \leq 2}=\frac{1}{\operatorname{det}\left(g_{i j}\right)}\left(\begin{array}{cc}
1+f_{y}^{2}+g_{y}^{2} & -f_{x} f_{y}-g_{x} g_{y} \\
-f_{x} f_{y}-g_{x} g_{y} & 1+f_{x}^{2}+g_{x}^{2}
\end{array}\right) .
$$

Moreover, the metric on the normal bundle $T^{\perp} \Sigma$ is

$$
\left(g_{\alpha \beta}\right)_{3 \leq \alpha, \beta \leq 4}=\left(\begin{array}{ll}
1+f_{x}^{2}+f_{y}^{2} & f_{x} g_{x}+f_{y} g_{y} \\
f_{x} g_{x}+f_{y} g_{y} & 1+g_{x}^{2}+g_{y}^{2}
\end{array}\right)
$$

and the inverse matrix is

$$
\left(g^{\alpha \beta}\right)_{3 \leq \alpha, \beta \leq 4}=\frac{1}{\operatorname{det}\left(g_{\alpha \beta}\right)}\left(\begin{array}{cc}
1+g_{x}^{2}+g_{y}^{2} & -f_{x} g_{x}-f_{y} g_{y} \\
-f_{x} g_{x}-f_{y} g_{y} & 1+f_{x}^{2}+f_{y}^{2}
\end{array}\right) .
$$

By a direct computation, we have

$$
\operatorname{det}\left(g_{i j}\right)=1+f_{x}^{2}+f_{y}^{2}+g_{x}^{2}+g_{y}^{2}+\left(f_{x} g_{y}-f_{y} g_{x}\right)^{2}
$$


and

$$
\operatorname{det}\left(g_{\alpha \beta}\right)=1+f_{x}^{2}+f_{y}^{2}+g_{x}^{2}+g_{y}^{2}+\left(f_{x} g_{y}-f_{y} g_{x}\right)^{2}=\operatorname{det}\left(g_{i j}\right) .
$$

From now on, we denote

$$
\operatorname{det}(g)=\operatorname{det}\left(g_{i j}\right)=\operatorname{det}\left(g_{\alpha \beta}\right) .
$$

Using our choice of $J$ and the basis, we have

$$
\begin{array}{ll}
J e_{1}=\left(0,1,-g_{x}, f_{x}\right), & J e_{2}=\left(-1,0,-g_{y}, f_{y}\right), \\
J v_{3}=\left(f_{y},-f_{x}, 0,1\right), & J v_{4}=\left(g_{y},-g_{x},-1,0\right) .
\end{array}
$$

It is easy to see that

$$
\cos \alpha=\frac{w\left(e_{1}, e_{2}\right)}{\sqrt{\operatorname{det}(g)}}=\frac{\left\langle J e_{1}, e_{2}\right\rangle}{\sqrt{\operatorname{det}(g)}}=\frac{1+f_{x} g_{y}-f_{y} g_{x}}{\sqrt{\operatorname{det}(g)}}
$$

and

$$
\sin ^{2} \alpha=\frac{\operatorname{det}(g)-\left\langle J e_{1}, e_{2}\right\rangle^{2}}{\operatorname{det}(g)}=\frac{\left(f_{x}-g_{y}\right)^{2}+\left(f_{y}+g_{x}\right)^{2}}{\operatorname{det}(g)} .
$$

We now express the Euler-Lagrangian equation (2.2) of $L$ explicitly. One checks that

$$
\begin{aligned}
\nabla \cos \alpha & =g^{i j}\left\langle\nabla \cos \alpha, e_{i}\right\rangle e_{j} \\
& =\left(g^{11} \frac{\partial \cos \alpha}{\partial x}+g^{12} \frac{\partial \cos \alpha}{\partial y}\right) e_{1}+\left(g^{12} \frac{\partial \cos \alpha}{\partial x}+g^{22} \frac{\partial \cos \alpha}{\partial y}\right) e_{2} \\
& =: A e_{1}+B e_{2} .
\end{aligned}
$$

Note that

$$
\begin{aligned}
& \left(J e_{1}\right)^{\top}=g^{i j}\left\langle J e_{1}, e_{i}\right\rangle e_{j}=g^{2 j}\left\langle J e_{1}, e_{2}\right\rangle e_{j}=\left\langle J e_{1}, e_{2}\right\rangle\left(g^{21} e_{1}+g^{22} e_{2}\right), \\
& \left(J e_{2}\right)^{\top}=g^{i j}\left\langle J e_{2}, e_{i}\right\rangle e_{j}=g^{1 j}\left\langle J e_{2}, e_{1}\right\rangle e_{j}=-\left\langle J e_{1}, e_{2}\right\rangle\left(g^{11} e_{1}+g^{12} e_{2}\right) .
\end{aligned}
$$

We obtain

$$
\begin{aligned}
(J \nabla \cos \alpha)^{\top} & =A\left(J e_{1}\right)^{\top}+B\left(J e_{2}\right)^{\top} \\
& =\left\langle J e_{1}, e_{2}\right\rangle\left[A\left(g^{21} e_{1}+g^{22} e_{2}\right)-B\left(g^{11} e_{1}+g^{12} e_{2}\right)\right] \\
& \left.=\left\langle J e_{1}, e_{2}\right\rangle\left[\left(A g^{21}-B g^{11}\right) e_{1}+\left(A g^{22}-B g^{12}\right) e_{2}\right)\right] .
\end{aligned}
$$

Moreover,

$$
\begin{aligned}
\left(J e_{1}\right)^{\perp} & =g^{\alpha \beta}\left\langle J e_{1}, v_{\alpha}\right\rangle v_{\beta} \\
& =\left(g^{33}\left\langle J e_{1}, v_{3}\right\rangle+g^{43}\left\langle J e_{1}, v_{4}\right\rangle\right) v_{3}+\left(g^{34}\left\langle J e_{1}, v_{3}\right\rangle+g^{44}\left\langle J e_{1}, v_{4}\right\rangle\right) v_{4} \\
\left(J e_{2}\right)^{\perp} & =g^{\alpha \beta}\left\langle J e_{2}, v_{\alpha}\right\rangle v_{\beta} \\
& =\left(g^{33}\left\langle J e_{2}, v_{3}\right\rangle+g^{43}\left\langle J e_{2}, v_{4}\right\rangle\right) v_{3}+\left(g^{34}\left\langle J e_{2}, v_{3}\right\rangle+g^{44}\left\langle J e_{2}, v_{4}\right\rangle\right) v_{4}
\end{aligned}
$$


It follows that

$$
\begin{aligned}
\left(J(J \nabla \cos \alpha)^{\top}\right)^{\perp}= & \left\langle J e_{1}, e_{2}\right\rangle\left[\left(A g^{21}-B g^{11}\right)\left(J e_{1}\right)^{\perp}+\left(A g^{22}-B g^{12}\right)\left(J e_{2}\right)^{\perp}\right] \\
= & \left\langle J e_{1}, e_{2}\right\rangle\left\{( A g ^ { 2 1 } - B g ^ { 1 1 } ) \left[\left(g^{33}\left\langle J e_{1}, v_{3}\right\rangle+g^{43}\left\langle J e_{1}, v_{4}\right\rangle\right) v_{3}\right.\right. \\
& \left.+\left(g^{34}\left\langle J e_{1}, v_{3}\right\rangle+g^{44}\left\langle J e_{1}, v_{4}\right\rangle\right) v_{4}\right] \\
& +\left(A g^{22}-B g^{12}\right)\left[\left(g^{33}\left\langle J e_{2}, v_{3}\right\rangle+g^{43}\left\langle J e_{2}, v_{4}\right\rangle\right) v_{3}\right. \\
& \left.\left.+\left(g^{34}\left\langle J e_{2}, v_{3}\right\rangle+g^{44}\left\langle J e_{2}, v_{4}\right\rangle\right) v_{4}\right]\right\} \\
= & \left\langle J e_{1}, e_{2}\right\rangle\left\{\left[\left(A g^{21}-B g^{11}\right)\left(g^{33}\left\langle J e_{1}, v_{3}\right\rangle+g^{43}\left\langle J e_{1}, v_{4}\right\rangle\right)\right.\right. \\
& \left.+\left(A g^{22}-B g^{12}\right)\left(g^{33}\left\langle J e_{2}, v_{3}\right\rangle+g^{43}\left\langle J e_{2}, v_{4}\right\rangle\right)\right] v_{3} \\
& +\left[\left(A g^{21}-B g^{11}\right)\left(g^{34}\left\langle J e_{1}, v_{3}\right\rangle+g^{44}\left\langle J e_{1}, v_{4}\right\rangle\right)\right. \\
& \left.\left.+\left(A g^{22}-B g^{12}\right)\left(g^{34}\left\langle J e_{2}, v_{3}\right\rangle+g^{44}\left\langle J e_{2}, v_{4}\right\rangle\right)\right] v_{4}\right\},
\end{aligned}
$$

and

$$
\begin{aligned}
H= & H^{\alpha} v_{\alpha}=g^{i j} h_{i j}^{\alpha} v_{\alpha}=g^{i j}\left(\frac{\partial^{2} F}{\partial x_{i} \partial x_{j}}\right)^{\perp} \\
= & g^{i j} g^{\alpha \beta}\left\langle\frac{\partial^{2} F}{\partial x_{i} \partial x_{j}}, v_{\alpha}\right\rangle v_{\beta} \\
= & \left(g^{33} g^{i j}\left\langle\frac{\partial^{2} F}{\partial x_{i} \partial x_{j}}, v_{3}\right\rangle+g^{43} g^{i j}\left\langle\frac{\partial^{2} F}{\partial x_{i} \partial x_{j}}, v_{4}\right\rangle\right) v_{3} \\
& +\left(g^{34} g^{i j}\left\langle\frac{\partial^{2} F}{\partial x_{i} \partial x_{j}}, v_{3}\right\rangle+g^{44} g^{i j}\left\langle\frac{\partial^{2} F}{\partial x_{i} \partial x_{j}}, v_{4}\right\rangle\right) v_{4} \\
= & {\left[g^{33}\left(g^{11}\left\langle\frac{\partial^{2} F}{\partial x^{2}}, v_{3}\right\rangle+2 g^{12}\left\langle\frac{\partial^{2} F}{\partial x \partial y}, v_{3}\right\rangle+g^{22}\left\langle\frac{\partial^{2} F}{\partial y^{2}}, v_{3}\right\rangle\right)\right.} \\
& \left.+g^{43}\left(g^{11}\left\langle\frac{\partial^{2} F}{\partial x^{2}}, v_{4}\right\rangle+2 g^{12}\left\langle\frac{\partial^{2} F}{\partial x \partial y}, v_{4}\right\rangle+g^{22}\left\langle\frac{\partial^{2} F}{\partial y^{2}}, v_{4}\right\rangle\right)\right] v_{3} \\
& +\left[g^{34}\left(g^{11}\left\langle\frac{\partial^{2} F}{\partial x^{2}}, v_{3}\right\rangle+2 g^{12}\left\langle\frac{\partial^{2} F}{\partial x \partial y}, v_{3}\right\rangle+g^{22}\left\langle\frac{\partial^{2} F}{\partial y^{2}}, v_{3}\right\rangle\right)\right. \\
& \left.+g^{44}\left(g^{11}\left\langle\frac{\partial^{2} F}{\partial x^{2}}, v_{4}\right\rangle+2 g^{12}\left\langle\frac{\partial^{2} F}{\partial x \partial y}, v_{4}\right\rangle+g^{22}\left\langle\frac{\partial^{2} F}{\partial y^{2}}, v_{4}\right\rangle\right)\right] v_{4} .
\end{aligned}
$$

By 2.7) and 2.8) we obtain

$$
\begin{aligned}
\cos ^{3} \alpha\left[g ^ { 3 3 } \left(g^{11}\left\langle\frac{\partial^{2} F}{\partial x^{2}}, v_{3}\right\rangle+\right.\right. & \left.2 g^{12}\left\langle\frac{\partial^{2} F}{\partial x \partial y}, v_{3}\right\rangle+g^{22}\left\langle\frac{\partial^{2} F}{\partial y^{2}}, v_{3}\right\rangle\right) \\
+ & \left.g^{43}\left(g^{11}\left\langle\frac{\partial^{2} F}{\partial x^{2}}, v_{4}\right\rangle+2 g^{12}\left\langle\frac{\partial^{2} F}{\partial x \partial y}, v_{4}\right\rangle+g^{22}\left\langle\frac{\partial^{2} F}{\partial y^{2}}, v_{4}\right\rangle\right)\right] \\
-\left\langle J e_{1}, e_{2}\right\rangle\left[\left(A g^{12}-\right.\right. & \left.B g^{11}\right)\left(g^{33}\left\langle J e_{1}, v_{3}\right\rangle+g^{43}\left\langle J e_{1}, v_{4}\right\rangle\right) \\
+ & \left.\left(A g^{22}-B g^{12}\right)\left(g^{33}\left\langle J e_{2}, v_{3}\right\rangle+g^{43}\left\langle J e_{2}, v_{4}\right\rangle\right)\right]=0
\end{aligned}
$$


and

$$
\begin{gathered}
\cos ^{3} \alpha\left[g^{34}\left(g^{11}\left\langle\frac{\partial^{2} F}{\partial x^{2}}, v_{3}\right\rangle+2 g^{12}\left\langle\frac{\partial^{2} F}{\partial x \partial y}, v_{3}\right\rangle+g^{22}\left\langle\frac{\partial^{2} F}{\partial y^{2}}, v_{3}\right\rangle\right)\right. \\
\left.+g^{44}\left(g^{11}\left\langle\frac{\partial^{2} F}{\partial x^{2}}, v_{4}\right\rangle+2 g^{12}\left\langle\frac{\partial^{2} F}{\partial x \partial y}, v_{4}\right\rangle+g^{22}\left\langle\frac{\partial^{2} F}{\partial y^{2}}, v_{4}\right\rangle\right)\right] \\
-\left\langle J e_{1}, e_{2}\right\rangle\left[\left(A g^{12}-B g^{11}\right)\left(g^{34}\left\langle J e_{1}, v_{3}\right\rangle+g^{44}\left\langle J e_{1}, v_{4}\right\rangle\right)\right. \\
\left.+\left(A g^{22}-B g^{12}\right)\left(g^{34}\left\langle J e_{2}, v_{3}\right\rangle+g^{44}\left\langle J e_{2}, v_{4}\right\rangle\right)\right]=0 .
\end{gathered}
$$

Set $c=1+f_{x} g_{y}-f_{y} g_{x}=\sqrt{\operatorname{det}(g)} \cos \alpha, a=f_{y}+g_{x}, b=f_{x}-g_{y}$. Recalling that

$$
F=(x, y, f(x, y), g(x, y)) \text {, }
$$

we have

$$
\begin{aligned}
\left\langle\frac{\partial^{2} F}{\partial x^{2}}, v_{3}\right\rangle & =f_{x x}, \quad\left\langle\frac{\partial^{2} F}{\partial x \partial y}, v_{3}\right\rangle=f_{x y}, \quad\left\langle\frac{\partial^{2} F}{\partial y^{2}}, v_{3}\right\rangle=f_{y y}, \\
\left\langle\frac{\partial^{2} F}{\partial x^{2}}, v_{4}\right\rangle & =g_{x x}, \quad\left\langle\frac{\partial^{2} F}{\partial x \partial y}, v_{4}\right\rangle=g_{x y}, \quad\left\langle\frac{\partial^{2} F}{\partial y^{2}}, v_{4}\right\rangle=g_{y y} .
\end{aligned}
$$

Moreover,

$$
\begin{array}{ll}
\left\langle J e_{1}, v_{3}\right\rangle=-f_{y}-g_{x}=-a, & \left\langle J e_{1}, v_{4}\right\rangle=f_{x}-g_{y}=b, \\
\left\langle J e_{2}, v_{3}\right\rangle=f_{x}-g_{y}=b, & \left\langle J e_{2}, v_{4}\right\rangle=f_{y}+g_{x}=a, \\
\left\langle J e_{1}, e_{2}\right\rangle=\sqrt{\operatorname{det}(g)} \cos \alpha=c . &
\end{array}
$$

Then equations 2.9 and 2.10 can be written as

$$
\begin{aligned}
& \frac{c^{2}}{(\sqrt{\operatorname{det}(g)})^{3}}\left[g^{33}\left(g^{11} f_{x x}+2 g^{12} f_{x y}+g^{22} f_{y y}\right)+g^{43}\left(g^{11} g_{x x}+2 g^{12} g_{x y}+g^{22} g_{y y}\right)\right] \\
& -\left\{\left[g^{12}\left(g^{34} b-g^{33} a\right)+g^{22}\left(g^{33} b+g^{34} a\right)\right] A-\left[g^{11}\left(g^{34} b-g^{33} a\right)+g^{12}\left(g^{33} b+g^{34} a\right)\right] B\right\}=0
\end{aligned}
$$

and

$$
\begin{aligned}
& \frac{c^{2}}{(\sqrt{\operatorname{det}(g)})^{3}}\left[g^{34}\left(g^{11} f_{x x}+2 g^{12} f_{x y}+g^{22} f_{y y}\right)+g^{44}\left(g^{11} g_{x x}+2 g^{12} g_{x y}+g^{22} g_{y y}\right)\right] \\
& -\left\{\left[g^{12}\left(g^{44} b-g^{34} a\right)+g^{22}\left(g^{34} b+g^{44} a\right)\right] A-\left[g^{11}\left(g^{44} b-g^{34} a\right)+g^{12}\left(g^{34} b+g^{44} a\right)\right] B\right\}=0 .
\end{aligned}
$$

Recalling that

$$
A=g^{11} \frac{\partial \cos \alpha}{\partial x}+g^{12} \frac{\partial \cos \alpha}{\partial y}, \quad B=g^{12} \frac{\partial \cos \alpha}{\partial x}+g^{22} \frac{\partial \cos \alpha}{\partial y},
$$


we have

$$
\begin{aligned}
& {\left[g^{12}\left(g^{34} b-g^{33} a\right)+g^{22}\left(g^{33} b+g^{34} a\right)\right] A-\left[g^{11}\left(g^{34} b-g^{33} a\right)+g^{12}\left(g^{33} b+g^{34} a\right)\right] B} \\
& =\left(g^{11} g^{22}-g^{12} g^{12}\right)\left(g^{33} b+g^{34} a\right) \frac{\partial \cos \alpha}{\partial x}+\left(g^{11} g^{22}-g^{12} g^{12}\right)\left(g^{33} a-g^{34} b\right) \frac{\partial \cos \alpha}{\partial y}
\end{aligned}
$$

and

$$
\begin{aligned}
& {\left[g^{12}\left(g^{44} b-g^{34} a\right)+g^{22}\left(g^{34} b+g^{44} a\right)\right] A-\left[g^{11}\left(g^{44} b-g^{34} a\right)+g^{12}\left(g^{34} b+g^{44} a\right)\right] B} \\
& =\left(g^{11} g^{22}-g^{12} g^{12}\right)\left(g^{34} b+g^{44} a\right) \frac{\partial \cos \alpha}{\partial x}+\left(g^{11} g^{22}-g^{12} g^{12}\right)\left(g^{34} a-g^{44} b\right) \frac{\partial \cos \alpha}{\partial y} .
\end{aligned}
$$

Therefore, equations 2.9 and 2.10 can be rewritten as

$$
\begin{aligned}
& \frac{c^{2}}{(\sqrt{\operatorname{det}(g)})^{3}}\left[g^{33}\left(g^{11} f_{x x}+2 g^{12} f_{x y}+g^{22} f_{y y}\right)+g^{43}\left(g^{11} g_{x x}+2 g^{12} g_{x y}+g^{22} g_{y y}\right)\right] \\
& -\left[\left(g^{11} g^{22}-g^{12} g^{12}\right)\left(g^{33} b+g^{34} a\right) \frac{\partial \cos \alpha}{\partial x}+\left(g^{11} g^{22}-g^{12} g^{12}\right)\left(g^{33} a-g^{34} b\right) \frac{\partial \cos \alpha}{\partial y}\right]=0
\end{aligned}
$$

and

$$
\begin{aligned}
& \frac{c^{2}}{(\sqrt{\operatorname{det}(g)})^{3}}\left[g^{34}\left(g^{11} f_{x x}+2 g^{12} f_{x y}+g^{22} f_{y y}\right)+g^{44}\left(g^{11} g_{x x}+2 g^{12} g_{x y}+g^{22} g_{y y}\right)\right] \\
& -\left[\left(g^{11} g^{22}-g^{12} g^{12}\right)\left(g^{34} b+g^{44} a\right) \frac{\partial \cos \alpha}{\partial x}+\left(g^{11} g^{22}-g^{12} g^{12}\right)\left(g^{34} a-g^{44} b\right) \frac{\partial \cos \alpha}{\partial y}\right]=0 .
\end{aligned}
$$

On the other hand, we have

$$
\begin{aligned}
\frac{\partial \cos \alpha}{\partial x}= & \frac{\partial}{\partial x}\left[\frac{1+f_{x} g_{y}-f_{y} g_{x}}{\sqrt{\operatorname{det}(g)}}\right] \\
= & \frac{1}{(\sqrt{\operatorname{det}(g)})^{3}}\left[f_{x x}\left(g_{y}-f_{x}+f_{y} g_{x} g_{y}+f_{x} f_{y} g_{y}+g_{y} f_{y}^{2}+g_{y} g_{x}^{2}+g_{y}^{3}-f_{x} g_{y}^{2}\right)\right. \\
& +f_{x y}\left(-g_{x}-f_{y}+f_{y} g_{x} g_{y}-f_{x} f_{y} g_{y}-g_{x} f_{x}^{2}-g_{x} g_{y}^{2}-g_{x}^{3}-f_{y} g_{x}^{2}\right) \\
& +g_{x x}\left(-g_{x}-f_{y}+f_{x} f_{y} g_{y}-f_{x} g_{x} g_{y}-f_{y} f_{x}^{2}-f_{y} g_{y}^{2}-f_{y}^{3}-g_{x} f_{y}^{2}\right) \\
& \left.+g_{x y}\left(f_{x}-g_{y}+f_{x} f_{y} g_{x}+f_{y} g_{x} g_{y}+f_{x} g_{x}^{2}+f_{x} f_{y}^{2}+f_{x}^{3}-g_{y} f_{x}^{2}\right)\right] \\
= & \frac{1}{(\sqrt{\operatorname{det}(g)})^{3}}\left[f_{x x}\left(g_{12} a-g_{22} b\right)+f_{x y}\left(g_{12} b-g_{11} a\right)\right. \\
& \left.+g_{x x}\left(-g_{22} a-g_{12} b\right)+g_{x y}\left(g_{11} b+g_{12} a\right)\right] .
\end{aligned}
$$


Similarly, one checks that

$$
\begin{aligned}
\frac{\partial \cos \alpha}{\partial y}= & \frac{1}{(\sqrt{\operatorname{det}(g)})^{3}}\left[f_{y y}\left(-g_{11} a+g_{12} b\right)+f_{x y}\left(g_{12} a-g_{22} b\right)\right. \\
& \left.+g_{y y}\left(g_{11} b+g_{12} a\right)+g_{x y}\left(-g_{22} a-g_{12} b\right)\right] .
\end{aligned}
$$

By 2.11)-2.14, we see that the system 2.2 is

$$
\begin{aligned}
& f_{x x}\left[g^{11} g^{33} c^{2}-\left(g^{11} g^{22}-g^{12} g^{12}\right)\left(g^{34} a+g^{33} b\right)\left(g_{12} a-g_{22} b\right)\right] \\
& +f_{x y}\left[2 g^{33} g^{12} c^{2}-\left(g^{11} g^{22}-g^{12} g^{12}\right)\left(g^{34} a+g^{33} b\right)\left(g_{12} b-g_{11} a\right)\right. \\
& \left.-\left(g^{11} g^{22}-g^{12} g^{12}\right)\left(g^{33} a-g^{34} b\right)\left(g_{12} a-g_{22} b\right)\right] \\
& +f_{y y}\left[g^{22} g^{33} c^{2}-\left(g^{11} g^{22}-g^{12} g^{12}\right)\left(g^{33} a-g^{34} b\right)\left(-g_{11} a+g_{12} b\right)\right] \\
& +g_{x x}\left[g^{11} g^{34} c^{2}-\left(g^{11} g^{22}-g^{12} g^{12}\right)\left(g^{34} a+g^{33} b\right)\left(-g_{22} a-g_{12} b\right)\right] \\
& +g_{x y}\left[2 g^{34} g^{12} c^{2}-\left(g^{11} g^{22}-g^{12} g^{12}\right)\left(g^{34} a+g^{33} b\right)\left(g_{11} b+g_{12} a\right)\right. \\
& -g_{y y}\left[g^{22} g^{34} c^{2}-\left(g^{11} g^{22}-g^{12} g^{12}\right)\left(g^{33} a-g^{12} g^{12}\right)\left(g^{33} a-g^{34} b\right)\left(-g_{22} a-g_{12} b\right)\right] \\
&
\end{aligned}
$$

and

$$
\begin{aligned}
& f_{x x}\left[g^{11} g^{34} c^{2}-\left(g^{11} g^{22}-g^{12} g^{12}\right)\left(g^{44} a+g^{34} b\right)\left(g_{12} a-g_{22} b\right)\right] \\
& +f_{x y}\left[2 g^{34} g^{12} c^{2}-\left(g^{11} g^{22}-g^{12} g^{12}\right)\left(g^{44} a+g^{34} b\right)\left(g_{12} b-g_{11} a\right)\right. \\
& \left.-\left(g^{11} g^{22}-g^{12} g^{12}\right)\left(g^{34} a-g^{44} b\right)\left(g_{12} a-g_{22} b\right)\right] \\
& +f_{y y}\left[g^{22} g^{34} c^{2}-\left(g^{11} g^{22}-g^{12} g^{12}\right)\left(g^{34} a-g^{44} b\right)\left(-g_{11} a+g_{12} b\right)\right] \\
& +g_{x x}\left[g^{11} g^{44} c^{2}-\left(g^{11} g^{22}-g^{12} g^{12}\right)\left(g^{44} a+g^{34} b\right)\left(-g_{22} a-g_{12} b\right)\right] \\
& +g_{x y}\left[2 g^{44} g^{12} c^{2}-\left(g^{11} g^{22}-g^{12} g^{12}\right)\left(g^{44} a+g^{34} b\right)\left(g_{11} b+g_{12} a\right)\right. \\
& -g_{y y}\left[g^{22} g^{44} c^{2}-\left(g^{11} g^{22}-g^{12} g^{12}\right)\left(g^{34} a-g^{44} b\right)\left(g_{11} b+g_{12} a\right)\right]=0 .
\end{aligned}
$$

For simplicity, we write the system as

$$
\begin{aligned}
& A_{11} f_{x x}+A_{12} f_{x y}+A_{22} f_{y y}+B_{11} g_{x x}+B_{12} g_{x y}+B_{22} g_{y y}=0, \\
& C_{11} f_{x x}+C_{12} f_{x y}+C_{22} f_{y y}+D_{11} g_{x x}+D_{12} g_{x y}+D_{22} g_{y y}=0,
\end{aligned}
$$

where $A_{i j}, B_{i j}, C_{i j}$ and $D_{i j}$ are defined clearly $(i, j=1,2)$. So the symbol of the system is

$$
\sigma:=\left(\begin{array}{cc}
A_{11} \xi^{2}+A_{12} \xi \eta+A_{22} \eta^{2} & B_{11} \xi^{2}+B_{12} \xi \eta+B_{22} \eta^{2} \\
C_{11} \xi^{2}+C_{12} \xi \eta+C_{22} \eta^{2} & D_{11} \xi^{2}+D_{12} \xi \eta+D_{22} \eta^{2}
\end{array}\right)
$$

A direct computation yields 


$$
\begin{aligned}
\operatorname{det}(\sigma)= & c^{4}\left(g^{33} g^{44}-g^{34} g^{34}\right)\left[\left(g^{11}\right)^{2} \xi^{4}+\left(g^{22}\right)^{2} \eta^{4}+4 g^{11} g^{12} \xi^{3} \eta+4 g^{12} g^{22} \xi \eta^{3}\right. \\
& \left.+2 g^{11} g^{22} \xi^{2} \eta^{2}+4\left(g^{12}\right)^{2} \xi^{2} \eta^{2}\right] \\
& +c^{2}\left(a^{2}+b^{2}\right)\left(g^{11} g^{22}-g^{12} g^{12}\right)\left(g^{33} g^{44}-g^{34} g^{34}\right)\left(g^{11} g_{22} \xi^{4}+g^{22} g_{11} \eta^{4}\right. \\
& +2 g^{12} g_{22} \xi^{3} \eta-2 g^{11} g_{12} \xi^{3} \eta+2 g^{12} g_{11} \xi \eta^{3}-2 g^{22} g_{12} \xi \eta^{3}+g^{11} g_{11} \xi^{2} \eta^{2} \\
& \left.+g^{22} g_{22} \xi^{2} \eta^{2}-4 g^{12} g_{12} \xi^{2} \eta^{2}\right) \\
= & c^{4}\left(g^{33} g^{44}-g^{34} g^{34}\right)\left(g^{11} \xi^{2}+2 g^{12} \xi \eta+g^{22} \eta^{2}\right)^{2} \\
& +\frac{c^{2}\left(a^{2}+b^{2}\right)}{\operatorname{det}(g)}\left(g^{11} g^{22}-g^{12} g^{12}\right)\left(g^{33} g^{44}-g^{34} g^{34}\right)\left(g_{22}^{2} \xi^{4}+g_{11}^{2} \eta^{4}\right. \\
& \left.-4 g_{12} g_{22} \xi^{3} \eta-4 g_{11} g_{12} \xi \eta^{3}+2 g_{11} g_{22} \xi^{2} \eta^{2}+4 g_{12}^{2} \xi^{2} \eta^{2}\right) \\
= & \frac{c^{4}}{\operatorname{det}(g)}\left(g^{11} \xi^{2}+2 g^{12} \xi \eta+g^{22} \eta^{2}\right)^{2}+\frac{c^{2}\left(a^{2}+b^{2}\right)}{(\operatorname{det}(g))^{3}}\left(g_{22} \xi^{2}-2 g_{12} \xi \eta+g_{11} \eta^{2}\right)^{2} \\
= & \frac{c^{2}\left(a^{2}+b^{2}+c^{2}\right)}{(\operatorname{det}(g))^{3}}\left(g_{22} \xi^{2}-2 g_{12} \xi \eta+g_{11} \eta^{2}\right)^{2} \\
= & \frac{c^{2}}{(\operatorname{det} g)^{2}}\left(g_{22} \xi^{2}-2 g_{12} \xi \eta+g_{11} \eta^{2}\right)^{2}=\frac{\cos ^{2} \alpha}{\operatorname{det} g}\left(g_{22} \xi^{2}-2 g_{12} \xi \eta+g_{11} \eta^{2}\right)^{2}
\end{aligned}
$$

Because $\left(g_{i j}\right)$ is positive definite and $\cos \alpha>0$, we see that

$$
\operatorname{det}(\sigma)>0 \quad \text { if }(\xi, \eta) \neq(0,0),
$$

which implies that equation (2.2) is elliptic. This proves the theorem.

\section{Equations of the Kähler angle of a symplectic critical surface}

In the following, we always choose the orthonormal basis $\left\{e_{1}, e_{2}, v_{3}, v_{4}\right\}$ on $M$ along $\Sigma$ such that $\left\{e_{1}, e_{2}\right\}$ is a basis of $\Sigma$ and $\omega$ takes the form (2.3), and the complex structure $J$ on $M$ takes the form (2.4).

Let $T \Sigma, N \Sigma$ be the tangent and normal bundles of $\Sigma$ in $M$ respectively. The second fundamental form $A: T \Sigma \times T \Sigma \rightarrow N \Sigma$ is defined by $A(X, Y)=\left(\bar{\nabla}_{X} Y\right)^{\perp}$ for any tangent vector fields $X, Y$. The operator $B: T \Sigma \times N \Sigma \rightarrow T \Sigma$ is defined by $B(X, N)=$ $\left(\bar{\nabla}_{X} N\right)^{\top}, N \in N \Sigma$. Here ()$^{\top}$ denotes the projection from $T M$ onto $T \Sigma$ and ()$^{\perp}$ denotes the projection onto $N \Sigma$. Evidently,

$$
\langle A(X, Y), N\rangle=-\langle Y, B(X, N)\rangle .
$$

Proposition 3.1. Let $M$ be a Kähler surface with Kähler form $\omega$ and $J$ be the complex structure compatible with $\omega$ on $M$. If $\Sigma$ is a symplectic surface which is smoothly immersed in $M$ with Kähler angle $\alpha$, then

$$
\begin{aligned}
\Delta \cos \alpha= & \cos \alpha\left(-\left|h_{1 k}^{3}-h_{2 k}^{4}\right|^{2}-\left|h_{1 k}^{4}+h_{2 k}^{3}\right|^{2}\right) \\
& +\sin \alpha\left(H_{, 1}^{4}+H_{, 2}^{3}\right)-\frac{\sin ^{2} \alpha}{\cos \alpha}\left(K_{1212}+K_{1234}\right) .
\end{aligned}
$$

where $K$ is the curvature operator of $M$ and $H_{, i}^{\alpha}=\left\langle\bar{\nabla}_{e_{i}}^{N} H, v_{\alpha}\right\rangle$. 
Proof. It is evident that

$$
\Delta \cos \alpha=\Delta \frac{\omega\left(e_{1}, e_{2}\right)}{\sqrt{\operatorname{det}(g)}}=\Delta \omega\left(e_{1}, e_{2}\right)-\frac{1}{2} \cos \alpha \Delta g_{i j} g^{i j}
$$

Using the property that $\bar{\nabla} \omega=0$, we obtain

$$
\begin{aligned}
\Delta \omega\left(e_{1}, e_{2}\right)= & \bar{\nabla}_{e_{k}} \bar{\nabla}_{e_{k}} \omega\left(e_{1}, e_{2}\right) \\
= & \omega\left(\bar{\nabla}_{e_{k}} \bar{\nabla}_{e_{k}} e_{1}, e_{2}\right)-\omega\left(\bar{\nabla}_{e_{k}} \bar{\nabla}_{e_{k}} e_{2}, e_{1}\right)+2 \omega\left(\bar{\nabla}_{e_{k}} e_{1}, \bar{\nabla}_{e_{k}} e_{2}\right) \\
= & \omega\left(\bar{\nabla}_{e_{k}}\left(\nabla_{e_{k}} e_{1}+A\left(e_{k}, e_{1}\right)\right), e_{2}\right)-\omega\left(\bar{\nabla}_{e_{k}}\left(\nabla_{e_{k}} e_{2}+A\left(e_{k}, e_{2}\right)\right), e_{1}\right) \\
& +2 \omega\left(A\left(e_{k}, e_{1}\right), A\left(e_{k}, e_{2}\right)\right) \\
= & \omega\left(\nabla_{e_{k}} \nabla_{e_{k}} e_{1}, e_{2}\right)+\omega\left(A\left(e_{k}, \nabla_{e_{k}} e_{1}\right), e_{2}\right)+\omega\left(\bar{\nabla}_{e_{k}} A\left(e_{k}, e_{1}\right), e_{2}\right) \\
& -\omega\left(\nabla_{e_{k}} \nabla_{e_{k}} e_{2}, e_{1}\right)+\omega\left(A\left(e_{k}, \nabla_{e_{k}} e_{2}\right), e_{1}\right)-\omega\left(\bar{\nabla}_{e_{k}} A\left(e_{k}, e_{2}\right), e_{1}\right) \\
& +2 \omega\left(A\left(e_{k}, e_{1}\right), A\left(e_{k}, e_{2}\right)\right) \\
= & \cos \alpha\left\langle\nabla_{e_{k}} \nabla_{e_{k}} e_{1}, e_{1}\right\rangle+\cos \alpha\left\langle\nabla_{e_{k}} \nabla_{e_{k}} e_{2}, e_{2}\right\rangle \\
& +\omega\left(\bar{\nabla}_{e_{k}} A\left(e_{k}, e_{1}\right), e_{2}\right)-\omega\left(\bar{\nabla}_{e_{k}} A\left(e_{k}, e_{2}\right), e_{1}\right) \\
& +2 \omega\left(A\left(e_{k}, e_{1}\right), A\left(e_{k}, e_{2}\right)\right) .
\end{aligned}
$$

It is not hard to check that

$$
\begin{aligned}
\frac{1}{2} \cos \alpha \Delta g_{i j} g^{i j} & =\frac{1}{2} \cos \alpha \Delta\left\langle e_{i}, e_{j}\right\rangle g^{i j}=\cos \alpha\left\langle\nabla_{e_{k}} \nabla_{e_{k}} e_{i}, e_{j}\right\rangle g^{i j} \\
& =\cos \alpha\left\langle\nabla_{e_{k}} \nabla_{e_{k}} e_{1}, e_{1}\right\rangle+\cos \alpha\left\langle\nabla_{e_{k}} \nabla_{e_{k}} e_{2}, e_{2}\right\rangle
\end{aligned}
$$

Putting the last two identities into (3.2) and using (2.3), we obtain

$$
\begin{aligned}
\Delta \cos \alpha= & \omega\left(\bar{\nabla}_{e_{k}} A\left(e_{k}, e_{1}\right), e_{2}\right)-\omega\left(\bar{\nabla}_{e_{k}} A\left(e_{k}, e_{2}\right), e_{1}\right) \\
& +2 \omega\left(A\left(e_{k}, e_{1}\right), A\left(e_{k}, e_{2}\right)\right) \\
= & \omega\left(\bar{\nabla}_{e_{k}}\left(h_{1 k}^{\alpha} v_{\alpha}\right), e_{2}\right)-\omega\left(\bar{\nabla}_{e_{k}}\left(h_{2 k}^{\alpha} v_{\alpha}\right), e_{1}\right)+2 \omega\left(h_{1 k}^{\alpha} v_{\alpha}, h_{2 k}^{\beta} v_{\beta}\right) \\
= & \omega\left(h_{k 1, k}^{\alpha} v_{\alpha}-h_{k 1}^{\alpha} h_{k l}^{\alpha} e_{l}, e_{2}\right)-\omega\left(h_{k 2, k}^{\alpha} v_{\alpha}-h_{k 2}^{\alpha} h_{k l}^{\alpha} e_{l}, e_{1}\right) \\
& +2 \omega\left(h_{1 k}^{\alpha} v_{\alpha}, h_{2 k}^{\beta} v_{\beta}\right) \\
= & \cos \alpha\left(-\left(h_{1 k}^{\alpha}\right)^{2}-\left(h_{2 k}^{\alpha}\right)^{2}+2 h_{1 k}^{3} h_{2 k}^{4}-2 h_{1 k}^{4} h_{2 k}^{3}\right) \\
& +\omega\left(h_{k k, 1}^{\alpha} v_{\alpha}-K_{\alpha k 1 k} v_{\alpha}, e_{2}\right)-\omega\left(h_{k k, 2}^{\alpha} v_{\alpha}-K_{\alpha k 2 k} v_{\alpha}, e_{1}\right) \\
= & \cos \alpha\left(-\left(h_{1 k}^{\alpha}\right)^{2}-\left(h_{2 k}^{\alpha}\right)^{2}+2 h_{1 k}^{3} h_{2 k}^{4}-2 h_{1 k}^{4} h_{2 k}^{3}\right) \\
& +\sin \alpha\left(H_{, 1}^{4}+H_{, 2}^{3}\right)-\sin \alpha\left(K_{4 k 1 k}+K_{3 k 2 k}\right) \\
= & \cos \alpha\left(-\left(h_{1 k}^{\alpha}\right)^{2}-\left(h_{2 k}^{\alpha}\right)^{2}+2 h_{1 k}^{3} h_{2 k}^{4}-2 h_{1 k}^{4} h_{2 k}^{3}\right) \\
& +\sin \alpha\left(H_{, 1}^{4}+H_{, 2}^{3}\right)-\sin \alpha\left(K_{1213}-K_{1224}\right) .
\end{aligned}
$$

Since $J$ is integrable, we have

$$
\begin{aligned}
K_{1212} & =K\left(e_{1}, e_{2}, J e_{1}, J e_{2}\right)=K\left(e_{1}, e_{2}, \cos \alpha e_{2}+\sin \alpha v_{3},-\cos \alpha e_{1}-\sin \alpha v_{4}\right) \\
& =\cos ^{2} \alpha K_{1212}-\sin ^{2} \alpha K_{1234}+\sin \alpha \cos \alpha\left(K_{1213}-K_{1224}\right) .
\end{aligned}
$$


We therefore obtain

$$
\sin \alpha\left(K_{1212}+K_{1234}\right)=\cos \alpha\left(K_{1213}-K_{1224}\right) .
$$

Then adding (3.4) into 3.3, we get

$$
\begin{aligned}
\Delta \cos \alpha= & \cos \alpha\left(-\left(h_{1 k}^{\alpha}\right)^{2}-\left(h_{2 k}^{\alpha}\right)^{2}+2 h_{1 k}^{3} h_{2 k}^{4}-2 h_{1 k}^{4} h_{2 k}^{3}\right) \\
& +\sin \alpha\left(H_{, 1}^{4}+H_{, 2}^{3}\right)-\frac{\sin ^{2} \alpha}{\cos \alpha}\left(K_{1212}+K_{1234}\right) .
\end{aligned}
$$

This proves the proposition.

Lemma 3.2. Suppose that $M$ is a Kähler surface. Then

$$
\operatorname{Ric}\left(J e_{1}, e_{2}\right)=\frac{1}{\cos \alpha}\left(K_{1212}+K_{1234}\right) .
$$

Proof. From the Bianchi identity we see that

$$
\begin{aligned}
\operatorname{Ric}\left(J e_{1}, e_{2}\right) & =\sum_{A=1}^{4} K\left(J e_{1}, e_{A}, e_{2}, e_{A}\right)=\sum_{A=1}^{4} K\left(J e_{1}, e_{A}, J e_{2}, J e_{A}\right) \\
& =-\sum_{A=1}^{4} K\left(J e_{1}, J e_{2}, J e_{A}, e_{A}\right)-\sum_{A=1}^{4} K\left(J e_{1}, J e_{A}, e_{A}, J e_{2}\right) \\
& =\sum_{A=1}^{4} K\left(e_{1}, e_{2}, e_{A}, J e_{A}\right)-\sum_{A=1}^{4} K\left(J e_{1}, J e_{A}, e_{2}, J e_{A}\right) \\
& =\sum_{A=1}^{4} K\left(e_{1}, e_{2}, e_{A}, J e_{A}\right)-\operatorname{Ric}\left(J e_{1}, e_{2}\right)
\end{aligned}
$$

where we have used the fact that $\left\{J e_{A}\right\}$ is also an orthonormal basis of $M$. Using 3.4 we get

$$
\begin{aligned}
\operatorname{Ric}\left(J e_{1}, e_{2}\right) & =\frac{1}{2} K\left(e_{1}, e_{2}, e_{A}, J e_{A}\right)=\sin \alpha\left(K_{1213}-K_{1224}\right)+\cos \alpha\left(K_{1212}+K_{1234}\right) \\
& =\frac{1}{\cos \alpha}\left(K_{1212}+K_{1234}\right) .
\end{aligned}
$$

Theorem 3.3. Suppose that $M$ is a Kähler surface and $\Sigma$ is a symplectic critical surface in $M$ with Kähler angle $\alpha$. Then $\cos \alpha$ satisfies

$$
\begin{aligned}
\Delta \cos \alpha & =\frac{3 \sin ^{2} \alpha-2}{\cos \alpha}|\nabla \alpha|^{2}-\cos \alpha \sin ^{2} \alpha\left(K_{1212}+K_{1234}\right) \\
& =\frac{3 \sin ^{2} \alpha-2}{\cos \alpha}|\nabla \alpha|^{2}-\cos ^{2} \alpha \sin ^{2} \alpha \operatorname{Ric}\left(J e_{1}, e_{2}\right) .
\end{aligned}
$$


Proof. If $\Sigma$ is a symplectic critical surface, then $H=\frac{\sin ^{2} \alpha}{\cos ^{2} \alpha} V$. It is easy to check that

$$
\begin{aligned}
\left(h_{1 k}^{3}-h_{2 k}^{4}\right)^{2}+\left(h_{1 k}^{4}+h_{2 k}^{3}\right)^{2} & =|H|^{2}+2|V|^{2}+2 H \cdot V=\left(\frac{\sin ^{4} \alpha}{\cos ^{4} \alpha}+2+2 \frac{\sin ^{2} \alpha}{\cos ^{2} \alpha}\right)|V|^{2} \\
& =\frac{1+\cos ^{4} \alpha}{\cos ^{4} \alpha}|\nabla \alpha|^{2}
\end{aligned}
$$

and

$$
\begin{aligned}
H_{, 1}^{4}+H_{, 2}^{3} & =\partial_{1}\left(\frac{\sin ^{2} \alpha}{\cos ^{2} \alpha} \partial_{1} \alpha\right)+\partial_{2}\left(\frac{\sin ^{2} \alpha}{\cos ^{2} \alpha} \partial_{2} \alpha\right)=\frac{\sin ^{2} \alpha}{\cos ^{2} \alpha} \Delta \alpha+2 \frac{\sin \alpha}{\cos ^{3} \alpha}|\nabla \alpha|^{2} \\
& =\frac{\sin \alpha}{\cos ^{2} \alpha}\left(-\Delta \cos \alpha-\cos \alpha|\nabla \alpha|^{2}\right)+2 \frac{\sin \alpha}{\cos ^{3} \alpha}|\nabla \alpha|^{2} .
\end{aligned}
$$

Putting these two equations into 3.1, we obtain

$$
\begin{aligned}
\Delta \cos \alpha= & \frac{-1-\cos ^{4} \alpha+2 \sin ^{2} \alpha-\sin ^{2} \alpha \cos ^{2} \alpha}{\cos ^{3} \alpha}|\nabla \alpha|^{2}-\frac{\sin ^{2} \alpha}{\cos ^{2} \alpha} \Delta \cos \alpha \\
& -\frac{\sin ^{2} \alpha}{\cos \alpha}\left(K_{1212}+K_{1234}\right) .
\end{aligned}
$$

Lemma 3.2 now yields (3.6).

Corollary 3.4. If $M$ is a Kähler-Einstein surface with scalar curvature $K_{0}$, and $\Sigma$ is a symplectic critical surface in $M$ with Kähler angle $\alpha$, we have

$$
\Delta \cos \alpha=\frac{3 \sin ^{2} \alpha-2}{\cos \alpha}|\nabla \alpha|^{2}-K_{0} \cos ^{3} \alpha \sin ^{2} \alpha .
$$

If, in addition, we assume that $K_{0} \geq 0$, then the symplectic critical surface $\Sigma$ is a holomorphic curve.

Proof. Suppose that $M$ is a Kähler-Einstein surface with scalar curvature $K_{0}$. Then we have

$$
\operatorname{Ric}\left(J e_{1}, e_{2}\right)=\cos \alpha K_{0},
$$

and the identity in the corollary follows. The second statement of the corollary follows from the maximum principle.

\section{Topology of the symplectic critical surfaces}

In this section we will analyze the topological properties of compact symplectic critical surfaces. At a point $p \in \Sigma$ with $\alpha(p)=0$ the tangent plane $T_{p} \Sigma$ of $M$ at $p$ is a complex line in $T_{F(p)} M$. So such a point is called a complex tangent point. We recall some equations obtained by Wolfson in [11] (see also [10]). We write the metric of $M$ as

$$
d s^{2}=\sum_{\beta=-1,1} \omega_{\beta} \bar{\omega}_{\beta} .
$$


The induced metric on $\Sigma$ can be written as

$$
d s_{\Sigma}^{2}=\phi \circ \bar{\phi},
$$

where $\phi$ is a complex valued 1-form defined up to a complex factor of norm one; furthermore, one can assume that

$$
\omega_{1}=\cos (\alpha / 2) \phi, \quad \omega_{-1}=\sin (\alpha / 2) \bar{\phi},
$$

where $\alpha$ is the Kähler angle.

Suppose that the complex second fundamental form of $\Sigma$ in $M$ is

$$
I I^{C}=a \phi^{2}+2 b \phi \bar{\phi}+c \bar{\phi}^{2} .
$$

Relative to the coframe field $\omega_{-1}, \omega_{1}$, there is a unitary connection $\omega_{\beta \gamma}$ which satisfies

$$
d \omega_{\beta}=\omega_{\beta \gamma} \wedge \omega_{\gamma}, \quad \omega_{\beta \gamma}+\omega_{\gamma \beta}=0 .
$$

We set

$$
\begin{aligned}
& \cos (\alpha / 2) \omega_{1}+\sin (\alpha / 2) \bar{\omega}_{-1}=\theta_{1}+\sqrt{-1} \theta_{2}, \\
& \sin (\alpha / 2) \omega_{1}-\cos (\alpha / 2) \bar{\omega}_{-1}=\theta_{3}+\sqrt{-1} \theta_{4},
\end{aligned}
$$

where $\theta_{k}, k=1, \ldots, 4$, is an orthonormal coframe of the Riemannian structure of $M$. So, along $\Sigma$ we have

$$
\sin (\alpha / 2) \omega_{1}-\cos (\alpha / 2) \bar{\omega}_{-1}=0 .
$$

It follows that ([10, (1.6)], [11, (2.18)])

$$
\frac{1}{2}\left(d \alpha+\sin \alpha\left(\omega_{-1-1}+\omega_{1 \overline{1}}\right)\right)=a \phi+b \bar{\phi} .
$$

The relation between the real second fundamental form and the complex second fundamental form is given in [11, Section 2],

$$
\left(\begin{array}{cc}
1 & 1 \\
\sqrt{-1} & -\sqrt{-1}
\end{array}\right)\left(\begin{array}{ll}
a & b \\
b & c
\end{array}\right)\left(\begin{array}{cc}
1 & \sqrt{-1} \\
1 & -\sqrt{-1}
\end{array}\right)=\left(\begin{array}{ll}
h_{11}^{3} & h_{12}^{3} \\
h_{12}^{3} & h_{22}^{3}
\end{array}\right)+\sqrt{-1}\left(\begin{array}{cc}
h_{11}^{4} & h_{12}^{4} \\
h_{12}^{4} & h_{22}^{4}
\end{array}\right) .
$$

We therefore have

$$
b=\frac{1}{4}\left(H^{3}+\sqrt{-1} H^{4}\right) .
$$

Using (4.1), 4.2] and 2.6, we see that on the symplectic critical surface we have

$$
\frac{\partial \sin \alpha}{\partial \bar{\zeta}}=(\sin \alpha) h,
$$

where $h$ is a smooth complex function, and $\zeta$ is a local complex coordinate on $\Sigma$. By Bers' result [2], we have 
Proposition 4.1. A nonholomorphic symplectic critical surface in a Kähler surface has at most finitely many complex points.

Set

$$
g(\alpha)=\ln \left(\sin ^{2} \alpha\right)
$$

Then using (3.6), we obtain

$$
\begin{aligned}
\Delta g(\alpha) & =-2|\nabla \alpha|^{2}-2 \frac{\cos \alpha}{\sin ^{2} \alpha} \Delta \cos \alpha-4 \frac{\cos ^{2} \alpha}{\sin ^{2} \alpha}|\nabla \alpha|^{2} \\
& =-4|\nabla \alpha|^{2}+2 \cos ^{2} \alpha\left(K_{1212}+K_{1234}\right) .
\end{aligned}
$$

This equation is valid away from the complex tangent points of $M$. By the Gauss equation and Ricci equation, we have

$$
R_{1212}=K_{1212}+h_{11}^{\alpha} h_{22}^{\alpha}-\left(h_{12}^{\alpha}\right)^{2}, \quad R_{1234}=K_{1234}+h_{1 k}^{3} h_{2 k}^{4}-h_{1 k}^{4} h_{2 k}^{3},
$$

where $R_{1212}$ is the curvature of $T \Sigma$ and $R_{1234}$ is the curvature of $N \Sigma$. Adding these two equations, we get

$$
\begin{aligned}
K_{1212}+K_{1234} & =R_{1212}+R_{1234}-\frac{1}{2}|H|^{2}+\frac{1}{2}\left(\left(h_{1 k}^{3}-h_{2 k}^{4}\right)^{2}+\left(h_{1 k}^{4}+h_{2 k}^{3}\right)^{2}\right) \\
& =R_{1212}+R_{1234}+|V|^{2}+H \cdot V=R_{1212}+R_{1234}+\frac{1}{\cos ^{2} \alpha}|\nabla \alpha|^{2} .
\end{aligned}
$$

Thus,

$$
R_{1212}+R_{1234}=\frac{1}{2} \frac{\Delta g(\alpha)}{\cos ^{2} \alpha}+\frac{|\nabla \alpha|^{2}}{\cos ^{2} \alpha} .
$$

Integrating the above equality over $\Sigma$ we have

$$
2 \pi(\chi(T \Sigma)+\chi(N \Sigma))=-2 \pi P-\int_{\Sigma} \frac{|\nabla \alpha|^{2}}{\cos ^{2} \alpha} d \mu_{\Sigma},
$$

where $\chi(T \Sigma)$ is the Euler characteristic of $\Sigma, \chi(N \Sigma)$ is the Euler characteristic of the normal bundle of $\Sigma$ in $M$, and $P$ is the sum of the orders of the complex tangent points. We have thus proved the following theorem.

Theorem 4.2. Let $\Sigma$ be a nonholomorphic symplectic critical surface in a Kähler surface $M$. Let $P$ denote the sum of the orders of the complex tangent points. Then

$$
\chi(T \Sigma)+\chi(N \Sigma)=-P-\frac{1}{2 \pi} \int_{\Sigma} \frac{|\nabla \alpha|^{2}}{\cos ^{2} \alpha} d \mu_{\Sigma} .
$$

Similarly, we can show 
Theorem 4.3. Let $\Sigma$ be a nonholomorphic symplectic critical surface in a Kähler surface $M$. Then

$$
F^{*} c_{1}(M)[\Sigma]=-P-\frac{1}{2 \pi} \int_{\Sigma} \frac{|\nabla \alpha|^{2}}{\cos ^{3} \alpha} d \mu_{\Sigma} .
$$

where $c_{1}(M)$ is the first Chern class of $M$ and $[\Sigma]$ is the fundamental homology class of $\Sigma$.

Proof. By (4.3) we also get

$$
\Delta g(\alpha)=-4|\nabla \alpha|^{2}+2 \cos ^{3} \alpha \operatorname{Ric}\left(J e_{1}, e_{2}\right) .
$$

Note that $\operatorname{Ric}\left(J e_{1}, e_{2}\right) d \mu_{\Sigma}$ is the pull back to $\Sigma$, by the immersion $F$, of the Ricci 2-form of $M$, i.e.,

$$
F^{*}\left(\operatorname{Ric}^{M}\right)=\operatorname{Ric}\left(J e_{1}, e_{2}\right) d \mu_{\Sigma} .
$$

Thus,

$$
F^{*}\left(\operatorname{Ric}^{M}\right)=\left(\frac{1}{2} \frac{\Delta g(\alpha)}{\cos ^{3} \alpha}+2 \frac{|\nabla \alpha|^{2}}{\cos ^{3} \alpha}\right) d \mu_{\Sigma}
$$

Integrating it over $\Sigma$, we obtain

$$
2 \pi F^{*} c_{1}(M)[\Sigma]=-2 \pi P-\int_{\Sigma} \frac{|\nabla \alpha|^{2}}{\cos ^{3} \alpha} d \mu_{\Sigma} .
$$

This proves the theorem.

Corollary 4.4. Suppose that $\Sigma$ is a symplectic critical surface in a Kähler surface M. Then

$$
\chi(T \Sigma)+\chi(N \Sigma)=F^{*} c_{1}(M)[N],
$$

and $\Sigma$ is a minimal surface with constant Kähler angle.

Proof. By Proposition 4.1. Webster's formula ([9, Proposition 1]), and Theorem 4.2, we have

$$
\int_{\Sigma} \frac{|\nabla \alpha|^{2}}{\cos ^{2} \alpha} d \mu_{\Sigma}=0
$$

That means $|\nabla \alpha|=0$, which implies that $\alpha \equiv$ constant. By [2.2], we see that $H=0$.

Remark 4.5. If $\Sigma$ is not compact, the corollary above is not true. In fact, the rotational symmetric surface $z=-\frac{1}{2} \log \left(x^{2}+y^{2}\right)$ in $\mathbb{R}^{3}$ considered as a surface in $\mathbb{C}^{2}$ is a symplectic critical surface which is not minimal.

Remark 4.6. By Lemma 4.1 and Remark 4.1 in [5], one checks that a minimal surface with constant Kähler angle is an infinitesimally holomorphic immersion ([6, p. 253, Definition]). So we have a variational approach for infinitesimally holomorphic immersions which are, in many cases, holomorphic. 
Let $g$ be the genus of $\Sigma, I_{\Sigma}$ the self-intersection number of $\Sigma$, and $D_{\Sigma}$ the number of double points of $\Sigma$. Then

$$
\chi(\Sigma)=2-2 g, \quad \chi(\nu)=I_{\Sigma}-2 D_{\Sigma} .
$$

Setting $c_{1}(\Sigma)=c_{1}(M)([\Sigma])$, we have

Corollary 4.7. Suppose that $\Sigma$ is a symplectic critical surface in a Kähler surface $M$. Then

$$
2-2 g-c_{1}(\Sigma)+I_{\Sigma}-2 D_{\Sigma}=0 .
$$

Proof. This follows easily from Theorems 4.2 and 4.3 . We omit the details.

\section{The gradient flow}

In this section we consider the gradient flow of the function $L$, i. e,

$$
\frac{d F}{d t}=\cos ^{2} \alpha H-\frac{1}{\cos \alpha}\left(J(J \nabla \cos \alpha)^{\top}\right)^{\perp} .
$$

We set

$$
f=\cos ^{2} \alpha H-\frac{1}{\cos \alpha}\left(J(J \nabla \cos \alpha)^{\top}\right)^{\perp} .
$$

It is clear that if $f=0$, then $\Sigma$ is a symplectic critical surface.

By the first variational formula of the functional $L$ (Theorem 2.2), we see that, along the flow,

$$
\begin{aligned}
\frac{d L}{d t} & =-2 \int_{\Sigma} \frac{1}{\cos ^{3} \alpha}\left|\cos ^{2} \alpha H-\frac{1}{\cos \alpha}\left(J(J \nabla \cos \alpha)^{\top}\right)^{\perp}\right|^{2} d \mu \\
& =-2 \int_{\Sigma} \frac{1}{\cos ^{3} \alpha}|f|^{2} d \mu .
\end{aligned}
$$

By Theorem 2.3, we know that 5.1 is a parabolic equation, and the short time existence can be shown by a standard argument. We set $\Sigma_{t}=F(\Sigma, t)$ with $\Sigma_{0}=\Sigma$.

Using the same local frame as in Section 2, we can write (5.1) as

$$
\frac{d F}{d t}=\cos ^{2} \alpha H-\sin ^{2} \alpha V \equiv f .
$$

We compute the evolution of the area element of $\Sigma_{t}$ along the flow.

Lemma 5.1.

$$
\frac{d}{d t} d \mu_{t}=\left(-\cos ^{2} \alpha|H|^{2}+\sin ^{2} \alpha V \cdot H\right) d \mu_{t} .
$$

Proof. It is easy to check that

$$
\begin{aligned}
\frac{\partial}{\partial t} g_{i j} & =\frac{\partial}{\partial t}\left\langle\frac{\partial F}{\partial x^{i}}, \frac{\partial F}{\partial x^{j}}\right\rangle=2\left\langle\frac{\partial f}{\partial x^{i}}, \frac{\partial F}{\partial x^{j}}\right\rangle=-2\left\langle f, \frac{\partial^{2} F}{\partial x^{i} \partial x^{j}}\right\rangle \\
& =2\left(-\cos ^{2} \alpha H^{\alpha}+\sin ^{2} \alpha V^{\alpha}\right) h_{i j}^{\alpha} .
\end{aligned}
$$


Here we have used the fact that $\nabla_{e_{i}} e_{j}=0$ at the fixed point. Therefore,

$$
\frac{d}{d t} d \mu_{t}=-f \cdot H d \mu_{t}=\left(-\cos ^{2} \alpha|H|^{2}+\sin ^{2} \alpha V \cdot H\right) d \mu_{t}
$$

Now we derive the evolution equation of $\cos \alpha$ along the flow 5.3 , which can be seen as a starting point for the study of the flow.

Theorem 5.2. Let $M$ be a Kähler surface. Assume that $\alpha$ is the Kähler angle of $\Sigma_{t}$ which evolves by the flow (5.3). Then $\cos \alpha$ satisfies the equation

$$
\begin{aligned}
\left(\frac{d}{d t}-\Delta\right) \cos \alpha= & \cos ^{3} \alpha\left(\left|h_{1 k}^{3}-h_{2 k}^{4}\right|^{2}+\left|h_{2 k}^{3}+h_{1 k}^{4}\right|^{2}\right)+\cos ^{2} \alpha \sin ^{2} \alpha \operatorname{Ric}\left(J e_{1}, e_{2}\right) \\
& +\cos \alpha \sin ^{2} \alpha|H|^{2}-\cos \alpha \sin ^{2} \alpha|V+H|^{2}
\end{aligned}
$$

where $\left\{e_{1}, e_{2}, v_{3}, v_{4}\right\}$ is an orthonormal basis of $T_{p} M$ such that $\omega, J$ take the form 2.3, 2.4.

Proof. Using the fact that $\bar{\nabla} \omega=0$ and Lemma 5.1, we have

$$
\begin{aligned}
\frac{\partial}{\partial t} \cos \alpha & =\frac{\partial}{\partial t} \frac{\omega\left(e_{1}, e_{2}\right)}{\sqrt{\operatorname{det}\left(g_{t}\right)}}=\omega\left(\bar{\nabla}_{e_{1}} f, e_{2}\right)-\omega\left(\bar{\nabla}_{e_{2}} f, e_{1}\right)-\frac{1}{2} \cos \alpha \frac{\partial}{\partial t} g_{i j} g^{i j} \\
& =\omega\left(\bar{\nabla}_{e_{1}} f, e_{2}\right)-\omega\left(\bar{\nabla}_{e_{2}} f, e_{1}\right)+\cos \alpha f \cdot H
\end{aligned}
$$

By breaking $\bar{\nabla}_{e_{1}} f$ and $\bar{\nabla}_{e_{2}} f$ into the normal and tangent parts, we get

$$
\begin{aligned}
\omega\left(\bar{\nabla}_{e_{1}} f, e_{2}\right)-\omega\left(\bar{\nabla}_{e_{2}} f, e_{1}\right) \\
=\omega\left(\bar{\nabla}_{e_{1}}^{N} f, e_{2}\right)-\omega\left(\bar{\nabla}_{e_{2}}^{N} f, e_{1}\right)+\omega\left(\bar{\nabla}_{e_{1}}^{T} f, e_{2}\right)-\omega\left(\bar{\nabla}_{e_{2}}^{N} f, e_{1}\right) \\
=\omega\left(\bar{\nabla}_{e_{1}}^{N} f, e_{2}\right)-\omega\left(\bar{\nabla}_{e_{2}}^{N} f, e_{1}\right)+\omega\left(B\left(e_{1}, f\right), e_{2}\right)-\omega\left(B\left(e_{2}, f\right), e_{1}\right) \\
=\omega\left(\bar{\nabla}_{e_{1}}^{N} f, e_{2}\right)-\omega\left(\bar{\nabla}_{e_{2}}^{N} f, e_{1}\right)+\cos \alpha\left(\left\langle B\left(e_{1}, f\right), e_{1}\right\rangle+\left\langle B\left(e_{2}, f\right), e_{2}\right\rangle\right) \\
=\omega\left(\bar{\nabla}_{e_{1}}^{N} f, e_{2}\right)-\omega\left(\bar{\nabla}_{e_{2}}^{N} f, e_{1}\right)-\cos \alpha f \cdot H .
\end{aligned}
$$

Combining these two identities we obtain

$$
\begin{aligned}
\frac{\partial}{\partial t} \cos \alpha= & \omega\left(\bar{\nabla}_{e_{1}}^{N} f, e_{2}\right)-\omega\left(\bar{\nabla}_{e_{2}}^{N} f, e_{1}\right) \\
= & \omega\left(\bar{\nabla}_{e_{1}}^{N}\left(\cos ^{2} \alpha H-\sin ^{2} \alpha V\right), e_{2}\right)-\omega\left(\bar{\nabla}_{e_{2}}^{N}\left(\cos ^{2} \alpha H-\sin ^{2} \alpha V\right), e_{1}\right) \\
= & \cos ^{2} \alpha\left(\omega\left(\bar{\nabla}_{e_{1}}^{N} H, e_{2}\right)-\omega\left(\bar{\nabla}_{e_{2}}^{N} H, e_{1}\right)\right) \\
& +\omega\left(\partial_{1} \cos ^{2} \alpha H, e_{2}\right)-\omega\left(\partial_{2} \cos ^{2} \alpha H, e_{1}\right) \\
& -\omega\left(\partial_{1} \sin ^{2} \alpha V, e_{2}\right)+\omega\left(\partial_{2} \sin ^{2} \alpha V, e_{1}\right) \\
& -\sin ^{2} \alpha\left(\omega\left(\bar{\nabla}_{e_{1}}^{N} V, e_{2}\right)-\omega\left(\bar{\nabla}_{e_{2}}^{N} V, e_{1}\right)\right) \\
=: & I+I I+I I I+I V .
\end{aligned}
$$


By Theorem 3.1 we have

$$
\begin{aligned}
I & =\cos ^{2} \alpha \sin \alpha\left(H_{, 1}^{4}-H_{, 2}^{3}\right) \\
& =\cos ^{2} \alpha\left(\Delta \cos \alpha+\cos \alpha\left|h_{1 k}^{3}-h_{2 k}^{4}\right|^{2}+\cos \alpha\left|h_{2 k}^{3}+h_{1 k}^{4}\right|^{2}+\sin ^{2} \alpha \operatorname{Ric}\left(J e_{1}, e_{2}\right)\right) .
\end{aligned}
$$

It is clear that

$$
\begin{aligned}
I I & =H^{4} \sin \alpha \partial_{1} \cos ^{2} \alpha+H^{3} \sin \alpha \partial_{2} \cos ^{2} \alpha=-2 \sin ^{2} \alpha \cos \alpha\left(H^{4} \partial_{1} \alpha+H^{3} \partial_{2} \alpha\right) \\
& =-2 \cos \alpha \sin ^{2} \alpha H \cdot V .
\end{aligned}
$$

From the definition of $V$, we can see that

$$
\begin{aligned}
I I I & =-\sin \alpha \partial_{1} \sin ^{2} \alpha \partial_{1} \alpha-\sin \alpha \partial_{2} \sin ^{2} \alpha \partial_{2} \alpha=-2 \sin ^{2} \alpha \cos \alpha|\nabla \alpha|^{2} \\
& =-2 \sin ^{2} \alpha \cos \alpha|V|^{2} .
\end{aligned}
$$

Similarly, one obtains

$$
\begin{aligned}
I V & =-\sin ^{2} \alpha \omega\left(\bar{\nabla}_{e_{1}}^{N}\left(\partial_{2} \alpha v_{3}+\partial_{1} \alpha v_{4}\right), e_{2}\right)+\sin ^{2} \alpha \omega\left(\bar{\nabla}_{e_{2}}^{N}\left(\partial_{2} \alpha v_{3}+\partial_{1} \alpha v_{4}\right), e_{1}\right) \\
& =-\sin ^{2} \alpha\left(\omega\left(\partial_{1} \partial_{1} \alpha v_{4}, e_{2}\right)-\omega\left(\partial_{2} \partial_{2} \alpha v_{3}, e_{1}\right)\right)=-\sin ^{3} \alpha \Delta \alpha \\
& =\sin ^{2} \alpha \Delta \cos \alpha+\sin ^{2} \alpha \cos \alpha|\nabla \alpha|^{2}=\sin ^{2} \alpha \Delta \cos \alpha+\sin ^{2} \alpha \cos \alpha|V|^{2} .
\end{aligned}
$$

Putting these equations into (5.6, we obtain

$$
\begin{aligned}
\frac{\partial}{\partial t} \cos \alpha= & \Delta \cos \alpha+\cos ^{3} \alpha\left|h_{1 k}^{3}-h_{2 k}^{4}\right|^{2}+\cos ^{3} \alpha\left|h_{2 k}^{3}+h_{1 k}^{4}\right|^{2} \\
& +\cos ^{2} \alpha \sin ^{2} \alpha \operatorname{Ric}\left(J e_{1}, e_{2}\right)-2 \cos \alpha \sin ^{2} \alpha H \cdot V-\cos \alpha \sin ^{2} \alpha|V|^{2} .
\end{aligned}
$$

This proves the theorem.

Theorem 5.3. Let $M$ be a Kähler-Einstein surface with scalar curvature $K_{0}$. Assume that $\alpha$ is the Kähler angle of $\Sigma_{t}$ which evolves by the flow (5.3). Then $\cos \alpha$ satisfies the equation

$$
\begin{aligned}
\left(\frac{d}{d t}-\Delta\right) \cos \alpha= & \cos ^{3} \alpha\left(\left|h_{1 k}^{3}-h_{2 k}^{4}\right|^{2}+\left|h_{2 k}^{3}+h_{1 k}^{4}\right|^{2}\right)+K_{0} \cos ^{3} \alpha \sin ^{2} \alpha \\
& +\cos \alpha \sin ^{2} \alpha|H|^{2}-\cos \alpha \sin ^{2} \alpha|V+H|^{2},
\end{aligned}
$$

where $\left\{e_{1}, e_{2}, v_{3}, v_{4}\right\}$ is an orthonormal basis of $T_{p} M$ such that $\omega, J$ take the form 2.3 , 2.4. Consequently, if $\Sigma$ is symplectic, then along the flow (5.3), at each time $t, \Sigma_{t}$ is symplectic.

Acknowledgments. This work was carried out when the first author was a post-doctor at the Abdus Salam ICTP. She thanks the center for their hospitality. The authors thank the referee for his helpful comments, and Prof. M. Micallef for explaining Webster's formula to them. The authors also thank Dr. Sun Jun for improving the computation in the proof of Theorem 2.3

This research was partially supported by NSFC. 


\section{References}

[1] Arezzo, C.: Minimal surfaces and deformations of holomorphic curves in Kähler-Einstein manifolds. Ann. Scuola Norm. Sup. Pisa Cl. Sci. 29, 473-481 (2000) Zbl 1010.58006 MR 1784183

[2] Bers, L.: An outline of the theory of pseudo-analytic functions. Bull. Amer. Math. Soc. 62, 291-331 (1956) Zbl 0072.07703 MR 0081936

[3] Chen, J., Tian, G.: Minimal surfaces in Riemannian 4-manifolds. Geom. Funct. Anal. 7, 873916 (1997) Zbl 0891.53042 MR 1475549

[4] Chen, S. S., Wolfson, J.: Minimal surfaces by moving frames. Amer. J. Math. 105, 59-83 (1983) Zbl 0521.53050 MR 0692106

[5] Han, X., Li, J.: Translating solitons to symplectic and Lagrangian mean curvature flows. Int. J. Math. 20, 443-458 (2009) Zbl 1171.53330 MR 2515049

[6] Micallef, M. J., Wolfson, J. G.: The second variation of area of minimal surfaces in fourmanifolds. Math. Ann. 295, 245-267 (1993) Zbl 0788.58016 |MR 1202392

[7] Simon, L.: Lectures on Geometric Measure Theory. Proc. Centre Math. Anal. 3, Australian National Univ. Press (1983) Zbl 0546.49019 MR 0756417

[8] Tian, G.: Symplectic isotopy in four dimensions. In: First International Congress of Chinese Mathematicians (Beijing, 1998), AMS/IP Stud. Adv. Math. 20, Amer. Math. Soc., Providence, RI, 143-147 (2001) Zbl 1054.53099 MR 1830170

[9] Webster, S. M.: Minimal surfaces in a Kähler surface. J. Differential Geom. 20, 463-470 (1984) Zbl 0561.53054 MR 0788290

[10] Wolfson, J.: Minimal surfaces in Kähler surfaces and Ricci curvature. J. Differential Geom. 29, 281-294 (1989) Zbl 0667.53044 MR 0982175

[11] Wolfson, J.: On minimal surfaces in a Kähler manifold of constant holomorphic sectional curvature. Trans. Amer. Math. Soc. 290, 627-646 (1985) Zbl 0573.53005 MR 0792816 